\title{
Effects of Lipid-Lowering Therapy with Rosuvastatin on Atherosclerotic Burden in Patients with Chronic Kidney Disease
}

\author{
Yukako Sawara, Takashi Takei, Keiko Uchida, Tetsuya Ogawa, \\ Takumi Yoshida, Ken Tsuchiya and Kosaku Nitta
}

\begin{abstract}
Objective Although previous studies suggest that treatment of dyslipidemia with statins reduces mortality and morbidity that are associated with cardiovascular disease, only a few studies have examined the efficacy of statins on atherosclerotic status in patients with chronic kidney disease (CKD).

Materials and Methods A 12-month, prospective, randomized study was designed to assess the efficacy of rosuvastatin in reducing circulating atherosclerotic parameters and renal function in patients with CKD. Thirty-eight patients with CKD and LDL cholesterol levels $\geqq 100 \mathrm{mg} / \mathrm{dL}$ were randomly assigned to receive $2.5 \mathrm{mg} / \mathrm{dL}$ rosuvastatin (group A, $\mathrm{n}=22$ ) or nonrosuavastatin therapy (group $\mathrm{B}, \mathrm{n}=16$ ). Lipid profile, estimated glomerular filtration rate (eGFR), high sensitivity C-reactive protein (hs-CRP), and intima-media thickness (IMT) were measured before and 12 months after rosuvastatin was added to the treatment.

Results Total cholesterol, low-density lipoprotein cholesterol, remnant-like particle-cholesterol and triglycerides were significantly reduced only in patients who received rosuvastatin. These parameters remained unchanged in patients who were not treated with rosuvastatin. eGFR was significantly increased from $50.7 \pm 18.7$ $\mathrm{mL} / \mathrm{min} / 1.73 \mathrm{~m}^{2}$ to $53.3 \pm 20.1 \mathrm{~mL} / \mathrm{min} / 1.73 \mathrm{~m}^{2}$ and a significant reduction of U-P was detected in group A patients $(0.17 \pm 0.29$ vs. $0.13 \pm 0.3 \mathrm{~g} / \mathrm{day} ; \mathrm{p}<0.01)$. In addition to the hypolipidemic effect, rosuvastatin treatment significantly reduced hs-CRP $(\mathrm{p}=0.0054)$. Moreover, maximal IMT at the baseline $(1.89 \pm 0.98 \mathrm{~mm}) \mathrm{de}-$ creased significantly to $1.75 \pm 0.87 \mathrm{~mm}$ at 12 months ( $\mathrm{p}=0.0231$ ).

Conclusion Rosuvastatin treatment, in addition to its beneficial effect on cholesterol levels, reduced maximal IMT and modified the inflammatory state of these patients.
\end{abstract}

Key words: chronic kidney disease, rosuvastatin, intima-media thickness, C-reactive protein, adiponectin

(Inter Med 47: 1505-1510, 2008)

(DOI: 10.2169/internalmedicine.47.1159)

\section{Introduction}

Patients with chronic kidney disease (CKD) have a markedly higher prevalence of cardiovascular disease (CVD) than the general population $(1,2)$. They have an altered plasma lipid profile that is characterized by increased levels of triglycerides, decreased levels of high-density cholesterol (HDL)-C, and no consistent change in low-density lipoprotein cholesterol (LDL)-C (3). Furthermore, dyslipidemia is considered a major cause of CVD in patients with CKD, and management of dyslipidemia plays an important role in the therapy of these patients (4).

It has been established that the benefits of hepatic hydroxymethyl glutaryl-CoA reductase inhibitors, or statins, in cardiovascular disease can be explained not only by their lipid-lowering potential but also by non-lipid-related mechanisms, the so-called "pleiotropic effects". Some of these beneficial effects are related to the anti-inflammatory properties of statins, which may contribute to this positive effect of statins on the incidence of cardiovascular events (5).

It was published recently that reducing the inflammatory

Department of Medicine, Kidney Center, Tokyo Women's Medical University, Tokyo

Received for publication March 26, 2008; Accepted for publication May 9, 2008

Correspondence to Dr. Kosaku Nitta, knitta@kc.twmu.ac.jp 
Table 1. Clinical Characteristics of Patients with CKD at Baseline

\begin{tabular}{lcc}
\hline Characteristics & Group A (n=22) & Group B (n=16) \\
\hline Sex (male/female) & $11 / 11$ & $9 / 7$ \\
Age (years) & $63.8 \pm 9.1$ & $67.0 \pm 7.9$ \\
BMI $\left(\mathrm{kg} / \mathrm{m}^{2}\right)$ & $23.1 \pm 2.5$ & $23.4 \pm 2.9$ \\
SBP $(\mathrm{mmHg})$ & $126.7 \pm 9.3$ & $128.3 \pm 8.1$ \\
DBP $(\mathrm{mmHg})$ & $76.7 \pm 8.2$ & $80.4 \pm 5.4$ \\
Total-C $(\mathrm{mg} / \mathrm{dL})$ & $228.5 \pm 47.6$ & $205.6 \pm 28.1$ \\
HDL-C (mg/dL) & $55.9 \pm 14.5$ & $54.9 \pm 10.6$ \\
LDL-C (mg/dL) & $130.3 \pm 24.3$ & $128.3 \pm 16.2$ \\
Triglyceride (mg/dL) & $177.1 \pm 73.2$ & $161.2 \pm 35.3$ \\
RLP-C (mg/dL) & $8.1 \pm 3.3$ & $5.4 \pm 1.9$ \\
eGFR (mL/min/1.73m $\left.{ }^{2}\right)$ & $50.7 \pm 18.7$ & $57.3 \pm 16.2 *$ \\
U-P (g/day) & $0.17 \pm 0.29$ & $0.12 \pm 0.3$ \\
FPG (mg/dL) & $97.1 \pm 10.9$ & $98.2 \pm 18.6$ \\
History of CVD & 7 & 6 \\
Medications (number) & & \\
ARBs & 6 & 5 \\
ACEIs & 2 & 2 \\
CCBs & 3 & 5 \\
$\beta$-blockers & 2 & \\
& & \\
\hline
\end{tabular}

BMI, body mass index; SBP, systolic blood pressure; DBP, diastolic blood pressure; Total-C, total cholesterol; HDL-C, high-density lipoprotein choleserol; RLP-C, remnant-like particle-cholesterol; eGFR, estimated glomerular filtration rate; U-P, urinary protein excretion; FPG, fasting plasma glucose; CVD, cardiovascular disease; ARB, angiotensin II receptor blocker; ACEI, angiotensin converting enzyme inhibitor; $\mathrm{CCB}$, calcium channel blocker ${ }^{*} \mathrm{p}<0.05$ vs. group A.

component of cardiovascular disease through the use of statin therapy improves the clinical outcome independent of the reduction in serum cholesterol levels (6). Although previous studies suggest that treatment of dyslipidemia with a statin reduces morbidity and mortality that are associated with $\operatorname{CVD}(7,8)$, only a few studies have examined the efficacy of statins on dyslipidemia (9) and atherosclerotic status in patients with CKD, and most of these studies were performed in patients who had end-stage renal disease (ESRD) and were on dialysis but not in nondialyzed patients. The present prospective study was designed to evaluate the inflammatory and anti-atherosclerotic responses of rosuvastatin in patients with CKD.

\section{Materials and Methods}

\section{Study population and treatment protocol}

Thirty-eight patients with CKD (stage 2 to 4 ) were enrolled. All received medical treatment at Department of Medicine, Kidney Center in Tokyo Women's Medical University. Written informed consent was obtained from all patients before the study. This study complies with the Declaration of Helsinki and was in agreement with the guidelines approved by the ethics committee at our institution. Inclusion criteria were CKD with an estimated GFR $<90 \mathrm{~mL} / \mathrm{min}$ and $\geqq 15 \mathrm{~mL} / \mathrm{min}$. Exclusion criteria were levels of fasting serum LDL-C of $<100 \mathrm{mg} / \mathrm{dL}$ and $>200 \mathrm{mg} / \mathrm{dL}$; the existence of liver, cardiovascular, infectious, or systemic disease unrelated to ESRD; and the history of vascular intervention, congestive heart failure, or myocardial infraction within the 3 month proceeding of the period of enrollment. No patient took lipid-lowering medication the 4 -week period before the study.

Fifty CKD patients were randomly selected for this study and divided into two groups ( 25 patients $\times 2$ groups). However, 3 patients in group A did not complete the study protocol and 9 patients in group B took different stains for treatment. All patients who met the eligibility criteria were randomly assigned to two groups. Group A included 22 patients who took rosuvastatin $(2.5 \mathrm{mg} /$ day for 12 months), and group B included 16 patients who did not take lipidlowering drugs. Patients who were already taking other drugs kept their pharmacologic schedule constant during the study. Most of patients were receiving anti-hypertensive agents including renin-angiotensin system blockers, and their dosages were not changed during rosuvastatin therapy as listed in Table 1. 
Table 2. Renal Function and Lipid Parameters in Both Groups of CKD

\begin{tabular}{lcccc}
\hline \multirow{2}{*}{ Parameters } & \multicolumn{2}{c}{ Group A } & \multicolumn{2}{c}{ Group B } \\
& Baseline & Month 12 & Baseline & Month 12 \\
& & & & \\
\hline eGFR (mL/min/1.73m $\left.{ }^{2}\right)$ & $50.7 \pm 18.7$ & $53.3 \pm 20.1^{*}$ & $57.3 \pm 16.2$ & $55.1 \pm 17.2$ \\
U-P (g/day) & $0.17 \pm 0.29$ & $0.13 \pm 0.3^{*}$ & $0.12 \pm 0.3$ & $0.17 \pm 0.4$ \\
Total-C (mg/dL) & $228.6 \pm 79.6$ & $179.2 \pm 26.1^{* *}$ & $205.0 \pm 28.1$ & $200.6 \pm 36.0$ \\
HDL-C (mg/dL) & $55.9 \pm 14.5$ & $61.6 \pm 14.0^{* *}$ & $54.9 \pm 10.6$ & $55.8 \pm 10.5$ \\
LDL-C (mg/dL) & $130.3 \pm 24.3$ & $98.6 \pm 12.9^{* *}$ & $128.3 \pm 16.2$ & $125.1 \pm 16.7$ \\
Triglyceride (mg/dL) & $177.1 \pm 73.2$ & $131.6 \pm 35.7^{* *}$ & $161.2 \pm 35.3$ & $159.3 \pm 28.5$ \\
RLP-C (mg/dL) & $8.1 \pm 3.3$ & $5.5 \pm 1.7^{* *}$ & $5.4 \pm 1.9$ & $5.4 \pm 1.8$ \\
& & & & \\
\hline
\end{tabular}

eGFR, estimated glomerular filtration rate; U-P, urinary protein excretion; Total-C, total cholesterol; HDL-C, high-density lipoprotein cholesterol; LDL-C, low-density lipoprotein cholesterol; RLP-C, remnant-like particle-cholesterol ${ }^{*} \mathrm{p}<0.01,{ }^{* *} \mathrm{p}<0.001$ vs. baseline values.

\section{Measurements of lipid profile and inflammatory markers}

Venous blood samples were obtained before and after 12 months of rosuvastatin treatment and stored frozen at $-80^{\circ} \mathrm{C}$. Serum creatinine, total cholesterol (TC), HDL-C, LDL-C, and serum triglyceride (TG) levels were determined by means of standard enzymatic methods. GFR was estimated by the MDRD formula as previously described (10). Urinary protein excretion (U-P) was determined by 24 hours. Plasma high-sensitivity C-reactive protein (hs-CRP) levels were measured with a latex-based turbidimetric immunoassay on a Hitachi analyzer (Sigma Chemical Co., St Louis, MO, USA). Fasting remnant-like particle-cholesterol (RLP-C) was also measured at the baseline and 12 months using a commercial kit (SRL Laboratory, Tokyo). Serum adiponectin (ADPN) was measured with a human ADPN ELISA kit (Otsuka Pharmaceuticals, Tokyo) as previously described (11).

\section{Measurement of IMT}

IMT of the common carotid arteries, a validated marker of generalized atherosclerosis, was determined by ultrasonographical B-mode imaging of the carotid artery performed using a high resolution real-time ultrasonograph with a 12-MHz in-line Sectascanner (SSA-390A; Toshiba, Tokyo) (12). For each patient, three measurements were performed on the bilateral carotid arteries and the averaged values of maximal IMT were considered for analysis. All measurements were made by the same examiner under blind conditions.

\section{Statistical analyses}

Data are expressed as means $+\mathrm{SD}$. We assessed differences between baseline and 12 month values using the $t$ test for paired data, univariate analysis for normally distributed variables and Mann-Whitney $\mathrm{U}$ test for skewed variables. The calculation was conducted by Stat View ver 5.0 (SAS, Cary, CA, USA) and statistical significance was assumed at $\mathrm{p}<0.05$.

Results

All patients from group A and group B completed the study. Table 1 lists baseline characteristics of the patients with CKD. Primary disease was nephrosclerosis in all patients studied. There were no statistically significant differences between the two study groups at baseline evaluation, while eGFR in group A was significantly lower $(50.7 \pm 18.7$ $\left.\mathrm{mL} / \mathrm{min} / 1.73 \mathrm{~m}^{2}\right)$ than those $\left(54.9 \pm 10.6 \mathrm{~mL} / \mathrm{min} / 1.73 \mathrm{~m}^{2}\right)$ in group B $(\mathrm{p}<0.05)$. History of CVD was recorded 7 patients in group A and 6 in group B. However, no patients had new CVD events during the study period. No significant difference was noted in medical treatment with anti-hypertensive agents including renin-angiotensin system blockers.

Comparison of average serum lipid levels at the baseline and 12 month endpoint showed the expected variations during rosuvastatin therapy in group A patients (Table 2): A significant reduction in serum levels of TC $(\mathrm{p}<0.001)$, LDL cholesterol $(\mathrm{p}<0.001)$, triglycerides $(\mathrm{p}=0.002)$, and RLP-C $(\mathrm{p}=0.002)$. Conversely, no significant variations in lipid levels appeared in group B patients (Table 2).

In addition to lipid-lowering effects of rosuvastatin, renal protective effects appeared in group A. eGFR was significantly increased from $50.7 \pm 18.7 \mathrm{~mL} / \mathrm{min} / 1.73 \mathrm{~m}^{2}$ to $53.3 \pm$ $20.1 \mathrm{~mL} / \mathrm{min} / 1.73 \mathrm{~m}^{2}$ in group A patients, while no significant changes in group B patients $(57.3 \pm 16.2$ vs. $55.1 \pm 17.2$ $\mathrm{mL} / \mathrm{min} / 1.73 \mathrm{~m}^{2}$ ). Moreover, a significant reduction of U-P was detected in group A patients $(0.17 \pm 0.29$ vs. $0.13 \pm 0.3 \mathrm{~g} /$ day; $\mathrm{p}<0.01$.

Serum hs-CRP levels decreased significantly from $893.3 \pm$ 893.6 to $496.4 \pm 341.5 \mathrm{ng} / \mathrm{mL}(\mathrm{p}<0.01)$ in group A patients. However, they remained stable in group B (Table 3). In addition, serum ADPN levels increased significantly from 9.7 \pm 5.3. to $10.5 \pm 4.6 \mu \mathrm{g} / \mathrm{mL}(\mathrm{p}<0.05)$ in group A patients. Finally, maximal IMT at the baseline $(1.89 \pm 0.98 \mathrm{~mm})$ decreased significantly to $1.75 \pm 0.87 \mathrm{~mm}$ at 12 months $(\mathrm{p}=$ 
Table 3. Inflammatory Parameters and IMT in Both Groups of CKD

\begin{tabular}{lcccc}
\hline \multirow{2}{*}{ Parameters } & \multicolumn{2}{c}{ Group A } & \multicolumn{2}{c}{ Group B } \\
& Baseline & Month 12 & Baseline & Month 12 \\
& & & & \\
\hline Hs-CRP $(\mathrm{ng} / \mathrm{mL})$ & $893.3 \pm 893.6$ & $496.4 \pm 341.5^{*}$ & $888.9 \pm 892.4$ & $844.4 \pm 700.8$ \\
Max IMT $(\mathrm{mm})$ & $1.89 \pm 0.98$ & $1.75 \pm 0.87^{*}$ & $2.17 \pm 1.05$ & $2.27 \pm 1.02^{*}$ \\
ADPN $(\mu \mathrm{g} / \mathrm{mL})$ & $9.7 \pm 5.3$ & $10.5 \pm 4.6^{*}$ & $8.9 \pm 3.9$ & $9.0 \pm 4.8$ \\
& & & & \\
\hline
\end{tabular}

Hs-CRP, high-sensitivity C-reactive protein; IMT, intima-media thickness; ADPN, adiponectin $* \mathrm{p}<0.05$ vs. baseline values. $* \mathrm{p}<0.05, * *_{\mathrm{p}}<0.01$ vs. baseline values.

A:

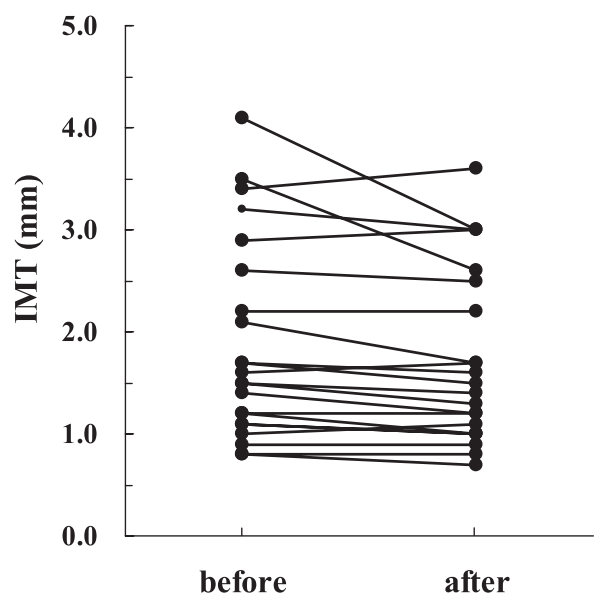

B:

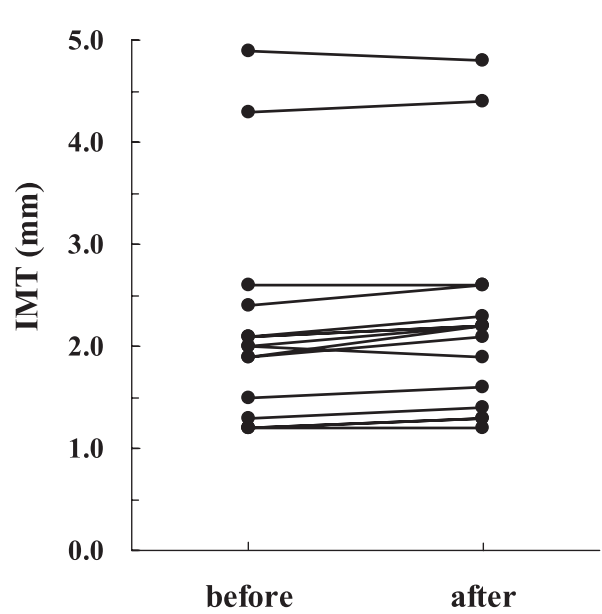

Figure 1. Changes in intima-media thickness (IMT) in 22 patients who took rosuvastatin (A) and in 16 patients who did not take lipid-lowering drugs $(B)$.

0.0231)(Fig. 1).

\section{Discussion}

Pleiotropic effects of statins have important clinical implications, independent of their lipid-lowering effects (13). Patients with CKD, even those in the early stages of the disorder, are at increased risk for CVD, and recent reviews suggested that oxidant stress and inflammation may be the primary mediators that explain the burden of CVD in patients with CKD (14). Although rosuvastatin reduced the incidence of cardiovascular events in patients who were on hemodialysis in a recently published large, prospective study (15), anti-atherosclerotic effects of this statin have not been explored in patients with CKD.

Our randomized, prospective study describes the impact of rosuvastatin on atherosclerotic parameters in nondialyzed patients with CKD. Serum levels of TC, LDL-C, RLP-C and TG were significantly reduced in patients who received rosuvastatin. In addition to the hypolipidemic effect, rosuvastatin significantly reduced hs-CRP and maximal IMT, suggesting that rosuvastatin treatment, in addition to its beneficial effect on serum lipid levels, reduced atherosclerotic state in these patients.
Slowing the progression of CKD by lipid-lowering agents could indirectly lead to reduction in CVD in these patients. The contribution of dyslipidemia to progression of CKD is supported by animal studies. Two meta-analyses $(16,17)$ and several post hoc analyses of large international studies $(7,18)$ provide further evidence that statins slow decline in GFR. However, the most recent systematic review which evaluated up to 40,000 participants revealed that statins only had a modest effect on slowing decline in GFR and was significantly only in those with known CVD (17). Two reports indicated that rosuvastatin treatment tended to preserve GFR in patients with $\operatorname{CKD}(19,20)$. Although previous reports suggest a benefit of statins in early stage CKD, similar results have not be replicated in more advanced (stages 4-5) CKD patients. This lack of efficacy in CKD may be related to delay in initiating therapy in patients who already have an atypically severe form of atherosclerosis (21).

A few recent studies described the impact of statins on serum CRP levels in patients with CKD. Panichi et al (9) described that simvastatin in commonly used dosages has in vitro and in vivo anti-inflammatory effect in patients with CKD. Recently, Goicoechea et al (22) described that atorvastatin treatments, in addition to its beneficial effect on lipid profile, improves the inflammatory state of patients 
with CKD without modifying fibrinolytic balance. These studies confirm the findings that patients with CKD have higher levels of inflammatory markers such as CRP than general population. As described in patients who had ESRD and were on hemodialysis therapy (23-25), statin treatment in group A patients significantly reduced inflammatory parameters.

Adiponectin (ADPN), the most abundant protein secreted from adipose tissue, possesses anti-atherogenic, antiinflammatory, and ant-diabetic effects (26). It is expected that the lipid-lowering drugs may be capable of increasing serum ADPN levels. The present study clearly shows that serum ADPN levels significantly increased in CKD patients treated with rosuvastatin. The present study suggested that anti-inflammatory effects of rosuvastatin may be related to the increase in ADPN levels. Iwashima et al (27) reported that renal function is a significant regulator of ADPN when categorized by CKD stage, whereas hypoadiponectinemia is a predictor of CVD, including recurrent ischemic heart disease. It is likely that the increased ADPN levels induced by rosuvastatin are related to a reduction of atherosclerotic burden in these patients.

\section{Study Limitations}

There are several limitations such as small sample size leading to inadequate assessment, short duration of therapy, and selection of a heterogeneous group of patients with CKD. A longer duration of the therapy warrants examination because the improvement in the atherosclerotic burden.

\section{Conclusion}

In nondialysis patients with CKD, treatment with rosuvastatin $(2.5 \mathrm{mg} /$ day $)$ for 12 months induced a lipid-lowering effect and a significant decrease in inflammatory parameters and IMT. Such evidence, similar to that observed in hemodialysis patients, could suggest a new therapeutic use of statins in the prevention of cardiovascular damage in patients with CKD.

\section{References}

1. US Renal Data System. Patient mortality and survival. Am J Kidney Dis 32 (Suppl 1): S69-S80, 1998.

2. Patient Registration Committee, Japanese Society for Dialysis Therapy. An overview of regular dialysis treatment in Japan as of 31 December 2003. Ther Apher Dial 9: 431-458, 2005.

3. Shoji T, Ishimura E, Inaba M, Tabata T, Nishizawa Y. Atherogenic lipoproteins in end-stage renal disease. Am J Kidney Dis 37: 484489, 2001.

4. National Kidney Foundation. K/DOQI: Treating dyslipidemia. Am J Kidney Dis 41: S39-S58, 2003.

5. Halcox JPJ, Deanfiel MB. Beyond the laboratory: Clinical implications for statin pleiotropy. Circulation 109 (Suppl II): II42-II48, 2004.

6. Nissen SE, Tuzcu M, Schoenhagen P, et al. Reversal of atherosclerosis with aggressive lipid lowering (REVERSAL) investigators: Statin therapy, LDL cholesterol, C-reactive protein, and coronary artery disease. N Engl J Med 352: 29-38, 2005.

7. Tonelli M, Isles C, Curhan GC, et al. Effect of pravastatin on cardiovascular events in people with chronic kidney disease. Circulation 110: 1557-1563, 2004.

8. Fathi R, Isbel N, Short L, Haluska B, Johnson S, Marwick TH. The effect of long-term aggressive lipid lowering on ischemic and atherosclerotic burden in patients with chronic kidney disease. Am J Kidney Dis 43: 45-52, 2004.

9. Panichi V, Paoletti S, Mantuano E, et al. In vivo and in vitro effects of simvastatin on inflammatory markers in pre-dialysis patients. Nephrol Dial Transplant 21: 337-344, 2006.

10. Imai E, Horio M, Nitta $\mathrm{K}$, et al. Modification of the modification of diet in renal disease (MDRD) study equation for Japan. Am J Kidney Dis 50: 927-937, 2007.

11. Takahashi M, Otsubo S, Uchida K, Yumura W, Nitta K. Association between serum adiponectin levels and arteriosclerosis in $\operatorname{IgA}$ nephropathy patients. Intern Med 46: 453-459, 2007.

12. Tanaka M, Babazono T, Takeda M, Iwamoto Y. Pulse pressure and chronic kidney disease in patients with type 2 diabetes. Hypertens Res 29: 345-352, 2006.

13. Epstein M, Campese VM. Pleiotropic effects of 3-hydroxy-3methylglutaryl coenzyme A reductase inhibitors on renal function. Am J Kidney Dis 45: 2-14, 2005.
14. Chan DT, Irish AB, Dogra GK, Watts GF. Dyslipidemia and cardiovascular disease: mechanisms, therapeutic opportunities and clinical trials. Atherosclerosis 196: 823-834, 2008.

15. Fellstrom B, Holdaas H, Jardinec AG, et al. Effect of rosuvastatin on outcomes in chronic haemodialysis patients: Baseline data from the AURORA study. Kidney Blood Press Res 30: 314-322, 2007.

16. Fried LF, Orchard TJ, Kasiske BL. Effect of lipid reduction on the progression of renal disease: a meta-analysis. Kidney Int 59: 260269, 2001.

17. Sandhu S, Wiebe N, Fried LF, Tonelli M. Statins for improving renal outcome: a meta-analysis. J Am Soc Nephrol 17: 2006-2016, 2006.

18. Collins R, Armitage J, Parish S, Sleigh P, Petro R. MRC/BHF Heart Protection Study of cholesterol-lowering with simvastatin in 5963 people with diabetes: a randomized placebo-controlled trial. Lancet 361: 2005-2016, 2003.

19. Vidt DG, Cressman MD, Harris S, et al. Rosuvastatin-induced arrest in progression of renal disease. Cardiology 102: 52-60, 2004.

20. Vidt DG, Harris S, McTaggart F, et al. Effect of short-term rosuvastatin treatment on estimated glomerular filtration rate. Am J Cardiol 97: 1602-1606, 2006.

21. Athyros VG, Mikhailidis DP, Papageorgiou AA, et al. The effect of statins versus untreated dyslipidaemia on renal function in patients with coronary heart disease. A subgroup analysis of the Greek atorvastatin and coronary heart disease evaluation (GREACE) study. J Clin Pathol 57: 728-734, 2004.

22. Goicoechea M, de Vinuesa SG, Lahera V, et al. Effects of atorvastatin on inflammatory and fibrinolytic parameters with chronic kidney disease. J Am Soc Nephrol 17: S231-S235, 2006.

23. Ichihara A, Hayashi $M$, Ryuzaki $M$, Handa M, Furukawa $T$, Saruta T. Fluvastatin prevents development of arterial stiffness in haemodialysis patients with type 2 diabetes mellitus. Nephrol Dail Transplant 17: 1513-1517, 2002.

24. Chang JW, Yang WS, Min WK, Lee SK, Park JS, Kim SB. Effects of simvastatin on high-sensitivity C-reactive proteins and serum albumin in hemodialysis patients. Am J Kidney Dis 39: 12131217, 2002.

25. Vernaglione L, Cristofano C, Muscogiuri P, Chimienti S. Does atorvastatin influence serum $\mathrm{C}$-reactive protein levels in patients 
Inter Med 47: 1505-1510, 2008 DOI: 10.2169/internalmedicine.47.1159

on long-term hemodialysis? Am J Kidney Dis 43: 471-478, 2004.

26. Matsuzawa Y, Funahashi T, Kihara S, Shimomura I. Adiponectin and metabolic syndrome. Arterioscler Thrombo Vasc Biol 24: 2933, 2004.
27. Iwashima $\mathrm{Y}$, Horio T, Kumada M, et al. Adiponectin and renal function, and implications as a risk of cardiovascular disease. Am J Cardiol 98: 1603-1608, 2006.

(C) 2008 The Japanese Society of Internal Medicine http://www.naika.or.jp/imindex.html 